Recherches sur les Encephalartos Lehm. (Zamiaceae) d'Afrique centrale 2. Apport de la morphologie foliaire dans la différenciation spécifique

Author(s): F. Malaisse, J. P. Sclavo and C. Crosiers

Source: Bulletin du Jardin botanique National de Belgique / Bulletin van de Nationale Plantentuin van België, Sep. 30, 1993, Vol. 62, No. 194 (Sep. 30, 1993), pp. 205-219

Published by: Botanic Garden Meise

Stable URL: https://www.jstor.org/stable/3668277

JSTOR is a not-for-profit service that helps scholars, researchers, and students discover, use, and build upon a wide range of content in a trusted digital archive. We use information technology and tools to increase productivity and facilitate new forms of scholarship. For more information about JSTOR, please contact support@jstor.org.

Your use of the JSTOR archive indicates your acceptance of the Terms \& Conditions of Use, available at https://about.jstor.org/terms 


\title{
Recherches sur les Encephalartos Lehm. (Zamiaceae) d'Afrique centrale 2. Apport de la morphologie foliaire dans la différenciation spécifique
}

par

\author{
F. Malaisse, J.P. Sclavo \& C. Crosiers (*)
}

\begin{abstract}
Summary. - The authors recall the strong interest of Melville's nomenclature for cones descriptions in Zamiaceae. They pinpoint the lack of such a framework for the description of leaves, while unfertile plants are common in the field. Therefore, they propose a schema involving some 22 characteristic points to be mentionned. An application is realized with the description of a new species from Zaire : Encephalartos schaijesii.
\end{abstract}

Au cours de leurs séjours en Afrique centrale, les auteurs (F.M. \& J.P.S.) ont eu l'occasion d'effectuer plusieurs observations originales relatives à des Encephalartos ainsi que de rassembler un abondant matériel qui est à présent en cours d'étude. Les résultats qui en découleront feront l'objet de diverses notes que nous poursuivons par des remarques quant aux apports de la morphologie foliaire en systématique ainsi que par la description d'une espèce nouvelle.

\section{Morphologie foliaire}

Les descriptions récentes d'Encephalartos, tant à l'occasion de publications de diagnose de taxons nouveaux (Devred 1959, Lisowski \& Malaisse 1971, Stevenson et al. 1990, Bamps \& Lisowski 1990) que lors d'édition de monographies (Giddy 1984, Goode 1989), utilisent fort à propos, en ce qui concerne les strobiles, la nomenclature proposée par Melville en 1957 (fig. 1).

(*) F. Malaisse \& C. Crosiers, U.R. Ecologie, Faculté des Sciences agronomiques, Passage des Déportés 2, B-5030 Gembloux (Belgique); J.P. Sclavo, Villa "La Finca", Plateau du Mont Boron, F-06300 Nice (France). - Manuscrit déposé le 7 octobre 1992. 
La description des feuilles ne bénéficie pas d'un canevas aussi rigoureux. Il convient en premier lieu de distinguer les cataphylles des feuilles. Pour ces dernières, les descriptions envisagent habituellement la forme de la feuille et sa longueur, moins régulièrement leur nombre; pour les folioles il est précisé leur nombre, leur disposition, la forme du limbe, l'ornementation du bord du limbe y compris la présence ou l'absence de spinules, sa (ou ses) couleur(s).

L'absence fréquente de strobiles renforce cependant l'intérêt d'une description minutieuse de la feuille, comme c'est déjà le cas pour les Dioon (De Luca et al. 1982). Cet intérêt ressort et s'affirme d'ailleurs à la lecture de travaux récents. En fait de nombreux auteurs utilisaient déjà, fort à propos, l'un ou l'autre caractère remarquable de la morphologie foliaire. Ainsi l'un de nous (Malaisse 1969) a montré l'apport du nombre de nervures de la foliole pour la distinction des Encephalartos du Zaïre. Giddy (1984) figure les folioles des 28 espèces sud-africaines dans son ouvrage "Cycads of South Africa». En outre, elle présente un tableau qui envisage cinq caractères foliaires, à savoir la longueur et la couleur de la feuille, la largeur de la foliole, la couleur du rachis, l'absence ou la transformation des folioles au niveau du pétiole et la présence éventuelle d'un «col» jaune à la base des folioles. Avec Goode (1989) apparait une information complémentaire, à savoir une représentation de la coupe transversale du rachis portant deux folioles.

En fait, un examen attentif de la feuille permet la distinction de caractères remarquables que nous passons ci-dessous en revue.

1. - En premier lieu, l'observation de la plante «in situ» permet de préciser l'ordre de grandeur du nombre de feuilles.

2. - L'examen du port permet d'évaluer l'angle réalisé par le rachis et l'axe du tronc. Cet angle (a) peut varier de 15 à $80^{\circ}$ (fig. $2 \mathrm{~A}$ ). Il varie avec l'âge des feuilles, les nouvelles couronnes fréquemment dressées s'étalant progressivement. Néanmoins il constitue un caractère spécifique lors du développement final des nouvelles feuilles.

3. - La forme générale de la feuille, sa longueur et sa couleur seront précisées. Les très jeunes feuilles de certaines espèces montrent une coloration différente de celle des feuilles adultes; elle sera également signalée.

Fig. 1. - Nomenclature de Melville utilisée pour la description des cônes de Zamiaceae. 


\section{vue du dessus de la bulla}

\section{face adaxiale}

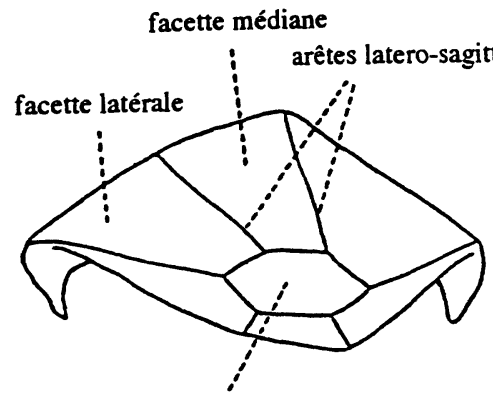

facette terminale arête sagittale supérieure

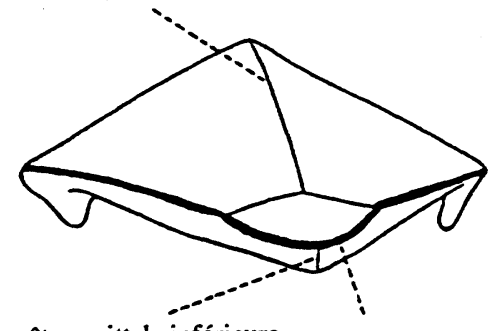

arête medio-latérale

face abaxiale

\section{vue latérale de l'écaille}
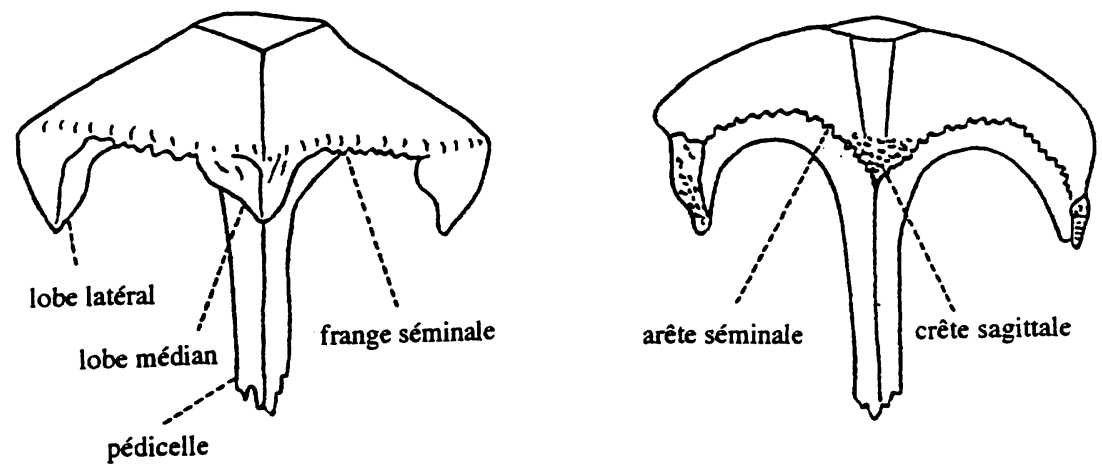

a. vue du côté adaxial

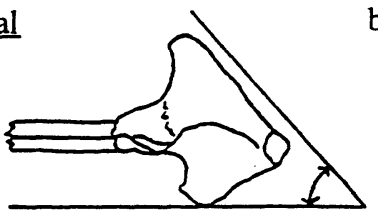

b. vue du côté abaxial

angle d'inclinaison de la bulla par rapport au pédicelle 
4. - Le pétiole et le rachis peuvent présenter une disposition rectiligne, ou au contraire avoir une extrémité courbée (concave ou convexe), voire encore une allure sigmoïde (fig. 2 B-C).

5. - Le pétiole et le rachis peuvent être glabres ou pubescents. Dans ce dernier cas la nature du trichome, sa couleur et sa disposition seront observées.

6. - La longueur du pétiole fournit une information utile.

7. - La nature des folioles vers la base de la feuille sera décrite: soit leur taille se réduit progressivement, soit elles se transforment en épines complexes ou simples.

8. - La présence ou l'absence d'un épaississement au niveau de l'insertion de la feuille sur le tronc sera examinée (fig. $4 \mathrm{~F}$ ).

9. - La section du rachis présente, selon les espèces, diverses silhouettes (fig. 3).

10. - Le nombre de folioles sera précisé.

11. - La forme, la longueur et la largeur de la foliole seront notées. Cette observation est réalisée à hauteur de la plus grande largeur de la feuille, habituellement au tiers supérieur. Le nombre de nervures doit encore être noté.

12. - La disposition des folioles sur le rachis peut être plus ou moins rapprochée ou écartée. Il est dès lors utile de mesurer la distance (d) séparant deux folioles successives (fig. $2 \mathrm{~J}$ ).

13. - Les folioles peuvent être entières ou diversement dentées. Le nombre de spinules sur chacun des bords est à préciser.

14. - Les folioles présentent fréquemment un sommet acuminé; l'acumen peut être situé dans l'axe de la foliole ou incliné par rapport à celui-ci, en position médiane ou latérale (fig. $2 \mathrm{~K}-\mathrm{L}$, fig. $5 \mathrm{C}$ ).

Fig. 2. - Caractères remarquables de la morphologie foliaire des Encephalartos (dessins C. Crosiers): A, représentation schématique de 2 valeurs de l'angle a réalisé par le rachis de la feuille et l'axe du tronc; B, E. horridus (Jacq.) Lehm., allure du pétiole et du rachis; C, E. ghellinckii Lem., idem; D-F, trois modes d'insertion des folioles sur le rachis (droit ou diversement oblique); G, E. sclavoi De Luca, Stevenson \& Moretti, recouvrement descendant des folioles [d'après Giddy (1984) modifié]; $\mathrm{H}$, E. longifolius (Jacq.) Lehm., recouvrement ascendant des folioles [d'après Giddy (1984) modifié]; I, angle b réalisé par les folioles entre elles; J, angle c compris entre l'axe du rachis et celui de la foliole et distance $\mathrm{d}$ séparant deux folioles successives; $\mathrm{K}$, E. schmitzii Malaisse, acumen situé dans l'axe de la foliole; L, E. engene-maraisii Verdoorn, acumen latéral et incliné par rapport à l'axe de la foliole. 
$-209-$
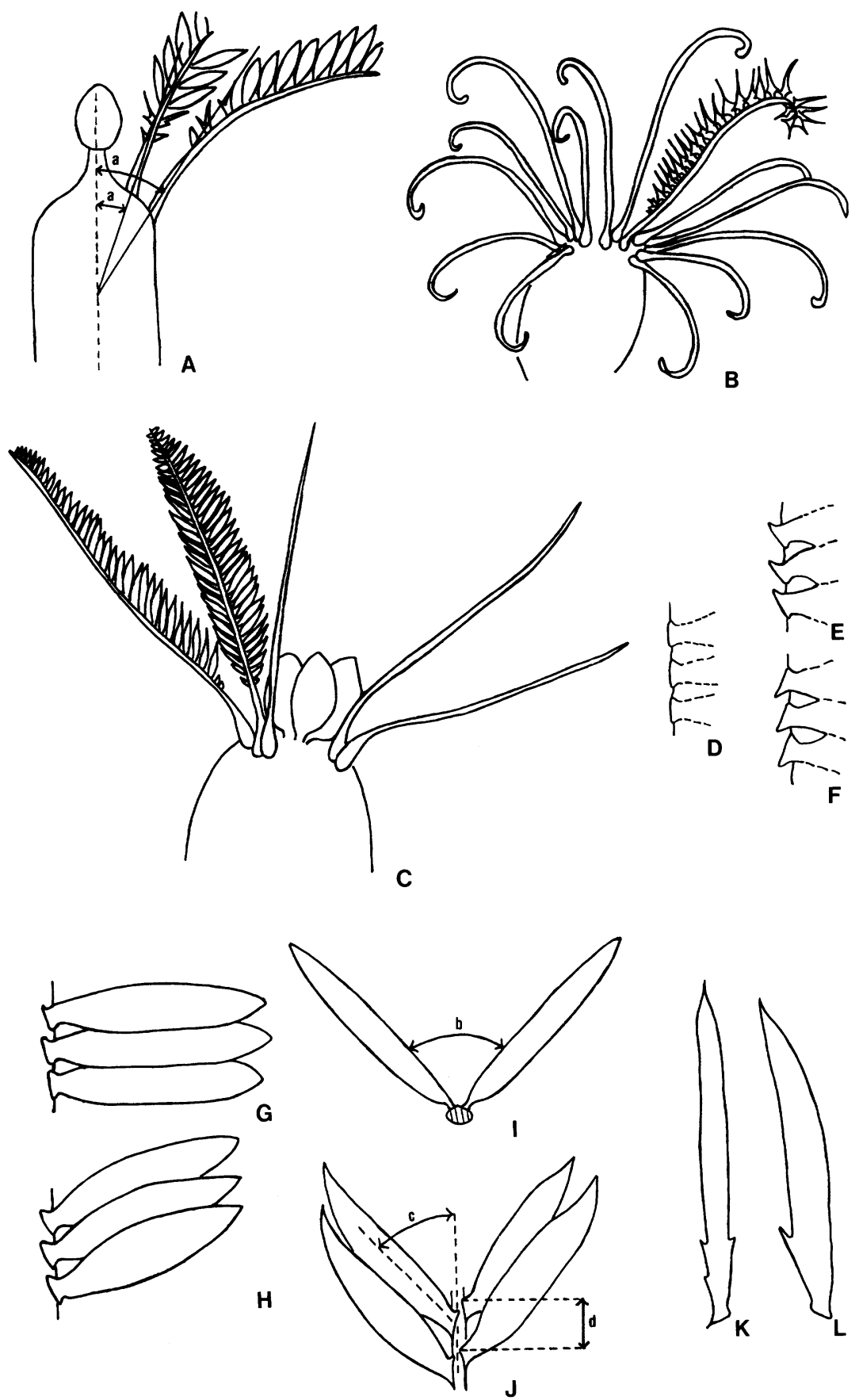

This content downloaded from

139.165.31.39 on Fri, 31 Dec 2021 08:21:35 UTC

All use subject to https://about.jstor.org/terms 


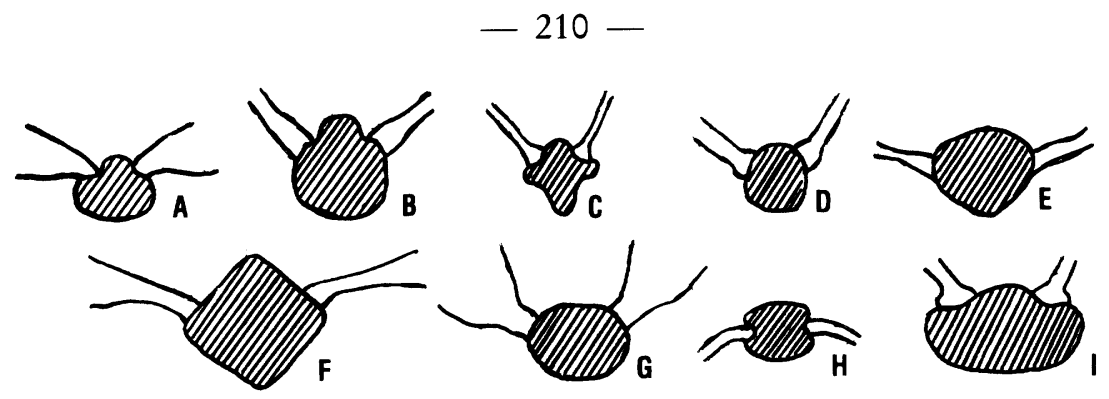

Fig. 3. - Allure de la section du rachis pour différents Encephalartos [dessins C. Crosiers d'après Goode (1989) modifié]: A, E. ngoyanus Verdoorn; B, E. turneri Lavranos \& Goode; C, E. schmitzii Malaisse; D, E. cupidus R.A. Dyer; E, E. barteri Carruth. ex Miq. ; F, E. transvenosus Stapf \& Davy; G, E. eugene-maraisii Verdoorn; $\mathrm{H}$, E. ghellinckii Lem.; I, E. delucanus Malaisse, Sclavo \& Crosiers.

15. - Les folioles (subopposées) réalisent entre elles un angle variable. Nous avons noté pour cet angle (b) des valeurs extrêmes de 40 et $270^{\circ}$ (fig. $2 \mathrm{I}$, fig. $4 \mathrm{~B}, \mathrm{H}$ ).

16. - L'axe des folioles, en absence de nervure principale, réalise avec l'axe du rachis un angle rarement droit. Cet angle (c) peut varier de 60 à $90^{\circ}$ (fig. $2 \mathrm{~J}$, fig. $5 \mathrm{~B}$ ).

17. - Les folioles peuvent ou non se recouvrir. Dans ce dernier cas, on distingue un recouvrement ascendant d'un recouvrement descendant (fig. 2 G-H, fig. 4 D,G). De plus, le segment de droite correspondant à l'insertion peut être parallèle à l'axe du rachis ou oblique (fig. 2 D-F, fig. 4 D-E).

18. - Les folioles s'insèrent sur le rachis à différents niveaux, notamment à la face supérieure ou latéralement (fig. 3).

19. - Une ligne longitudinale plus foncée joignant les insertions des folioles est parfois observée sur le rachis.

Fig. 4. - Morphologie foliaire de divers Encephalartos: A, E. longifolius (Jacq.) Lehm., allure générale, développement d'une nouvelle couronne de feuilles, port étalé $(\times 1 / 36) ; \mathrm{B}, E$. heenani R.A. Dyer, folioles réfléchies réalisant un angle b d'environ $270^{\circ}(\times 1 / 4) ; C$, E. laurentianus De Wild., allure générale, développement d'une nouvelle couronne de feuilles, port érigé $(\times 1 / 36) ; \mathrm{D}$, E. longifolius (Jacq.) Lehm., vue de détail permettant d'observer l'insertion des folioles sur le rachis, y compris la présence d'une zone jaunâtre ainsi que le recouvrement ascendant des folioles $(\times 2 / 3)$; $\mathrm{E}$, E. ituriensis Bamps \& Lisowski, vue de détail illustrant une insertion droite de la foliole sur le rachis, la présence d'une zone jaune et d'un étranglement à la base de la foliole $(\times 1) ; \mathrm{F}$, E. lehmannii Ecklon \& Zehyer ex Lehm., vue de l'épaississement à la base du pétiole $(\times 1 / 3)$; G, E. sclavoi De Luca, Stevenson $\&$ Moretti, vue de détail montrant un recouvrement descendant des folioles $(\times 1 / 5) ; \mathrm{H}$, E. longifolius (Jacq.) Lehm., folioles réalisant un angle $b$ d'environ $90^{\circ}(\times 1 / 6)$ (A, C-D, F, H: photographies C. Crosiers; B, E, G: photographies F. Malaisse). 

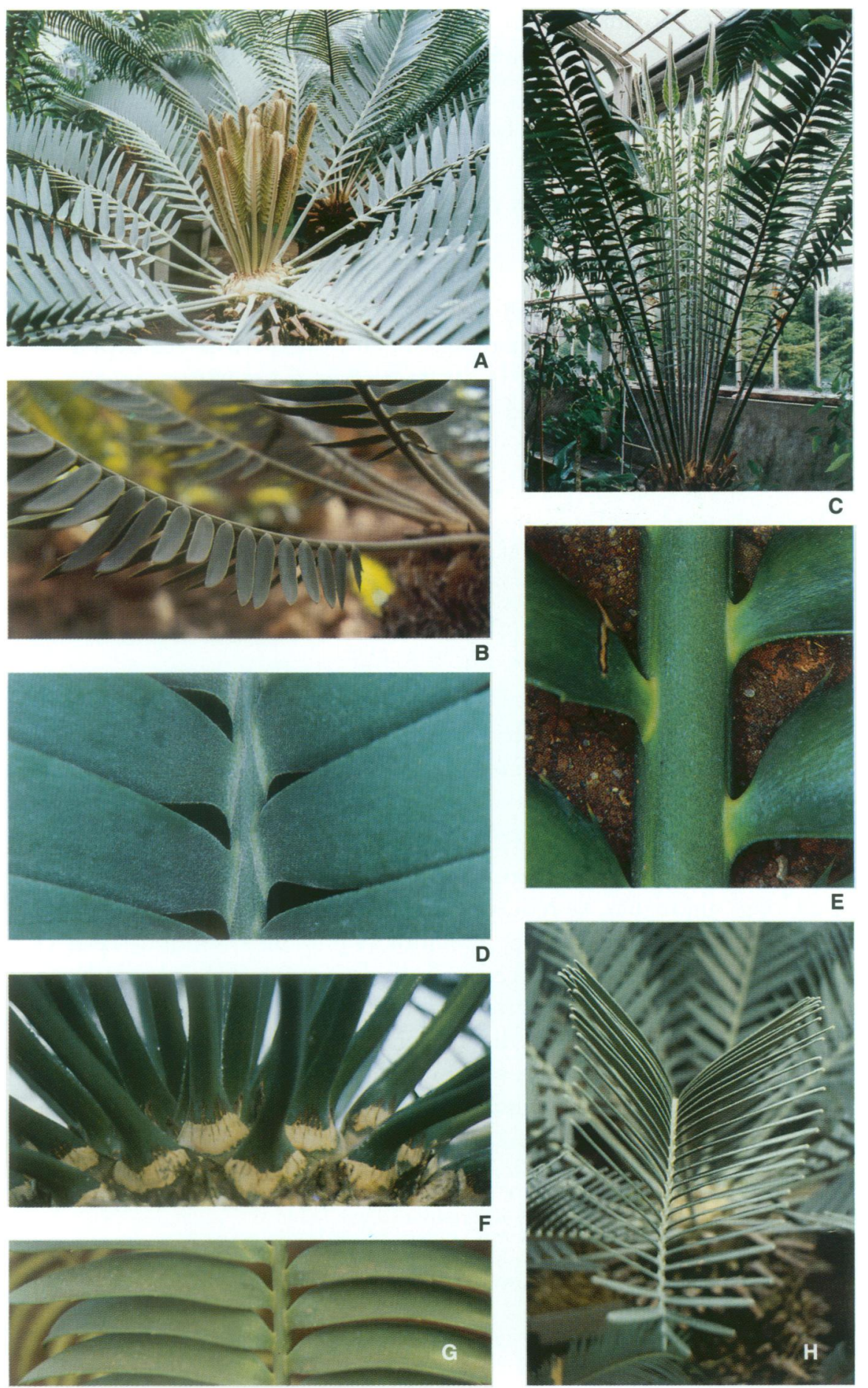

This content downloaded from

139.165.31.39 on Fri, 31 Dec 2021 08:21:35 UTC

All use subject to https://about.jstor.org/terms 
20. - A proximité de la base de la foliole un rétrécissement peut être présent ou absent (fig. $4 \mathrm{E}$ ). Il y a intérêt à préciser la valeur de sa largeur.

21. - L'insertion de la foliole est parfois soulignée par une zone de coloration différente et qui sera précisée (fig. 4 D-E).

22. - L'insertion de la foliole sur le rachis peut être (ou non) décurrente vers la base.

Il convient encore de signaler que, à côté de la morphologie de la feuille, les apports de l'anatomie foliaire ont parfois été exploités. Ainsi, Koeleman et al.(1981), sur la base de comparaison de coupes foliaires, ont établis une clef d'identification pour 28 espèces sud-africaines.

\section{Une espèce méconnue d'Encephalartos de Tanzanie}

Encephalartos delucanus Malaisse, Sclavo \& Crosiers, Ann. Gembloux 98 : 153 (1992). — Fig. 5 A-D,F.

Tronc dressé, pouvant atteindre $12 \mathrm{~cm}$ de hauteur (au dessus du sol), de $10-20 \mathrm{~cm}$ de diam. Cataphylle lancéolée, de $30-40 \mathrm{~mm}$ de long, $10 \mathrm{~mm}$ de large, à face adaxiale brune, glabre, à face abaxiale densément tomenteuse, à poils gris-beige.

Feuilles, 6-9, vertes, effectuant un angle (a) de $60-80^{\circ}$, de $50-65 \mathrm{~cm}$ de long, oblongues, se réduisant rapidement vers la base, recourbées au sommet; pétiole pouvant atteindre $45 \mathrm{~mm}$, de $7-8 \mathrm{~mm}$ de large et 6-7 mm d'épaisseur, pubescent, à poils de 3-4 $\mathrm{mm}$ de long, gris-beige et à poils de $1 \mathrm{~mm}$ de long brun-noir ; rachis pubescent à la base, glabre au sommet, orné de deux lignes vert sombre à la face supérieure; folioles (50-)66(-75), disposées sur la partie supérieure du rachis, imbriquées de façon descendante, réalisant un angle (b) de $45^{\circ}$ entre

Fig. 5. - Encephalartos delucanus et E. schaijesii Malaisse, Sclavo \& Crosiers: A, extrémité recourbée du rachis d'E. delucanus $(\times 1 / 2) ; B$, allure générale de folioles médianes d'E. delucanus $(\times 1 / 2) ; C$, extrémité d'une foliole d'E. delucanus $(\times 1) ; \mathrm{D}$, allure générale d'E. delucanus $(\times 1 / 16) ; \mathrm{E}, E$. schaijesii portant 2 cônes femelles, en culture dans un village zaïrois $(\times 1 / 36) ; \mathrm{F}$, base d'une feuille d'E. delucanus $(\times 1)$; (A-C, F : photographies F. Malaisse; D: photographie J.P. Sclavo; E : photographie A. Moretti). 

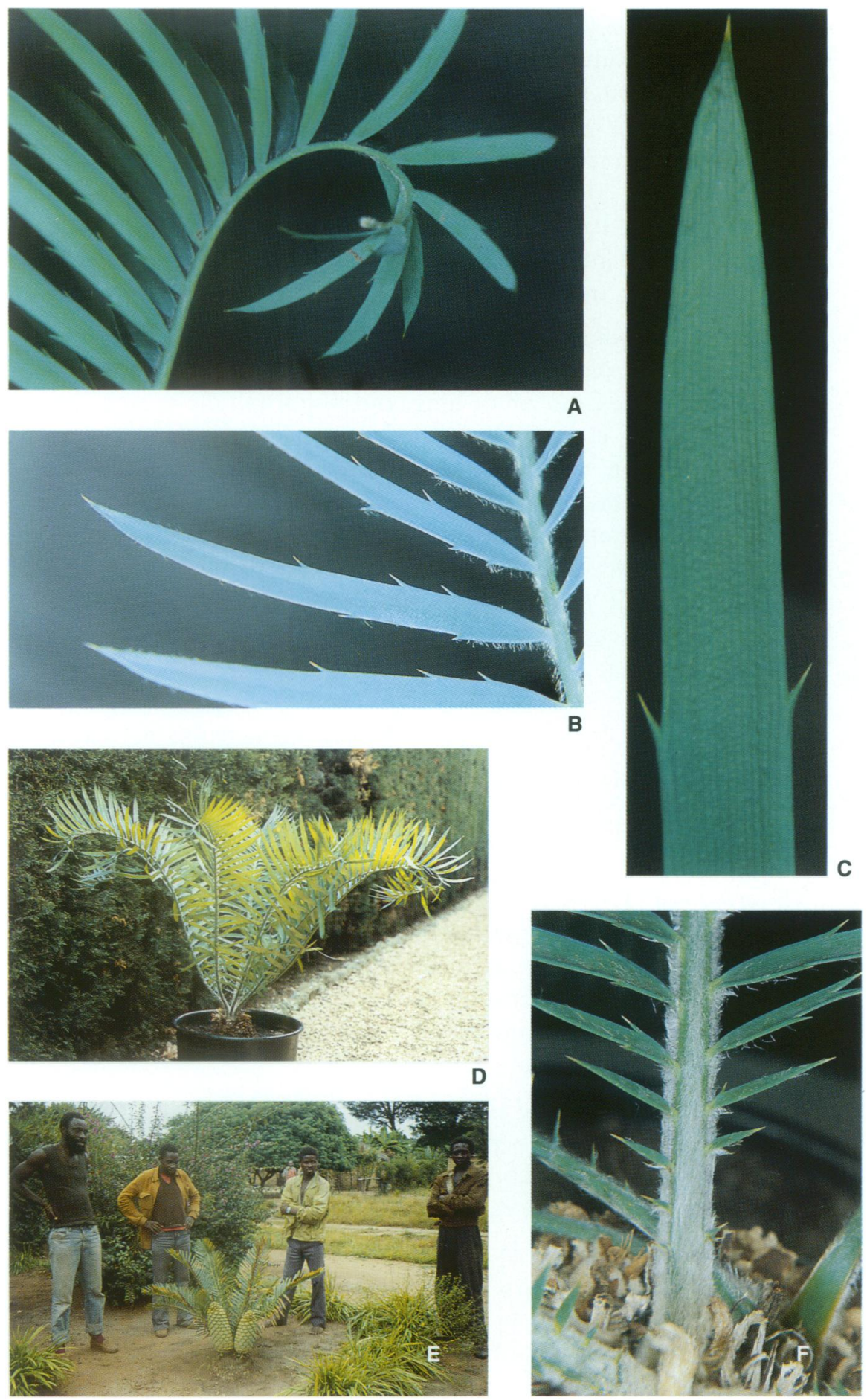

This content downloaded from

139.165.31.39 on Fri, 31 Dec 2021 08:21:35 UTC

All use subject to https://about.jstor.org/terms 
elles, et disposées selon un angle (c) de $70-75^{\circ}$ avec l'axe du rachis; folioles médianes subopposées à alternes, vertes, oblongues, 130-146 mm de long, (11-)12-14 mm de large, à sommet graduellement acuminé, piquant, à base jaune, rétrécie, de $4 \mathrm{~mm}$ de large et décurrente; 13-19 nervures parallèles; spinules latérales jaunes, 2-3(-4) au bord supérieur, (2-)3-4(-5) au bord inférieur, de 4-5 mm de long; spinule terminale produite dans l'axe de la foliole, $2-4 \mathrm{~mm}$ de long; folioles inférieures diminuant rapidement, 2-4 folioles bifurquées, de 2 à 6 folioles réduites et transformées en épines.

Strobile $\sigma^{\prime \prime}$ ellipsoïde à subcylindrique, jaune-verdâtre à l'état jeune, vert à l'état adulte, de $10-12 \mathrm{~cm}$ de long et de $2-3 \mathrm{~cm}$ de diam. à l'état sec, à pédoncule de $1-1,5 \mathrm{~cm}$ de long; microsporophylle moyenne de 12-14 mm de long et de 9-11 mm de large, inclinée par rapport à l' axe du cône selon un angle de $60-70^{\circ}$, courtement pédicellée, à pédicelle de 1-3 mm de long, à face adaxiale de la bulla concave, une facette terminale rhomboïdale, 2 facettes latérales trapézoïdales et une facette médiane rectangulaire, facette terminale parallèle à l'axe du cône; face abaxiale recouverte de sporanges, munies de 2 facettes latérales séparées entre elles par une crête médiane peu marquée. Strobile 9 inconnu.

Tanzanie : Western Province (T4), District Mpanda, Mgoga ( $\pm 5^{\circ} 45^{\prime} \mathrm{S}, 31^{\circ} 30^{\prime} \mathrm{E}$ ), env. $30 \mathrm{~km}$ sud de Katambike, au sud de la rivière Ugalla, savane boisée, Sclavo 180 (BR, O', holotype); Monts Kasima, au NE de Katuma (6 $6^{\circ} 19^{\prime} \mathrm{S}, 30^{\circ} 42^{\prime} \mathrm{E}$ ), savane boisée, Sclavo $20\left(\mathrm{BR}, \mathrm{O}^{\prime \prime}\right)$; Mont Sitebi $\left(6^{\circ} 06^{\prime} \mathrm{S}, 30^{\circ} 38^{\prime} \mathrm{E}\right)$, alt. $1950 \mathrm{~m}$, prairie ouverte, Kielland 21 (EA); Lugala Hills, alt. 1500 m, Kielland 22 (EA).

Observations: 1. - Cette nouvelle espèce correspond à l'Encephalartos sp. 'C' («Mpanda») de Heenan (1977:287). Les commentaires de Heenan se rapportaient au matériel récolté par Kielland dans le district de Mpanda, district d'où provient également l'holotype. Le matériel de Kielland a été examiné à Nairobi (EA) par l'un de nous (JPS).

2. - L'espèce est dédiée au Dr. P. De Luca, Professeur ordinaire de botanique au Département de Biologie végétale de l'Université Federico II de Naples et Directeur du Jardin Botanique de Naples, en hommage à ses travaux relatifs aux Cycadales.

3. - Kielland note que la plante est très rare. Au Mont Sitebi, il a observé deux bouquets d'une vingtaine d'individus distants de $300 \mathrm{~m}$. 
4. - E. delucanus appartient à un groupe d'espèces à tronc très court et feuilles de taille médiocre qui sont observées de l'Angola à la Tanzanie et auquel appartient E. poggei, E. schmitzii et E. marunguensis. Ces quatre espèces se distinguent aisément les unes des autres, notamment par leurs caractères foliaires.

\section{Une espèce nouvelle du Zaïre}

Suite à l'observation d'un pied transplanté dans un village (fig. $5 \mathrm{E}$ ), nous avions connaissance depuis plusieurs années de l'existence d'un Encephalartos dans les environs de Kolwezi au Shaba. Deux excursions ont permis de préciser la distribution de ce taxon et de réaliser son étude systématique. Il s'agit d'une espèce nouvelle que nous décrivons ci-dessous.

Encephalartos schaijesii Malaisse, Sclavo \& Crosiers sp. nov. fig. $5 \mathrm{E}$ et 6 .

Truncus erectus, ad $11-25 \mathrm{~cm}$ altus, $20-33 \mathrm{~cm}$ diam. Cataphylla lanceolata, 35-95 mm longa, $5 \mathrm{~mm}$ lata, pagina adaxiali brunnea glabraque, pagina abaxiali pilis lanatis stramineis. Folia 6-13, viridia vel leviter glauca, ad angulum (a) $25-50^{\circ}$ patentia, $76-118 \mathrm{~cm}$ longa, oblonga, ad basin abrupte attenuata; petiolus ad $20 \mathrm{~mm}$ longus, 8-10 $\mathrm{mm}$ latus, 7-7,5 $\mathrm{mm}$ crassus, pubescens, pilis 3-4 mm longis stramineis et pilis $1 \mathrm{~mm}$ longis brunneis; rachis ad basin pubescens, ad apicem glabra; foliola 96-117, ad rachidis partem superiorem disposita, ad rachidis axem parallela, haud imbricata, angulo (b) $70-80^{\circ}$, angulo (c) $70-75^{\circ}$; foliola mediana subopposita, oblonga, 125-167 mm longa, 14-18 mm lata, apice acuminata, pungentia, basi luteo-viridia, coarctata, 5-6 mm lata, ad basin decurrentia, folioli pilis petioli pilis similibus; foliola ad $38 \mathrm{~mm}$ (d) distantia; nervi 18-20; spinulae laterales luteo-virides, superne $0-1(-2)$ inferneque (0-1-)2-3, 2-4 $\mathrm{mm}$ longae; spina terminalis in folioli axe producta, $1-2 \mathrm{~mm}$ longa; foliola inferiora abrupte diminuta, 2-4 foliola bifurcata, 6-14 foliola infima in spinas mutata. Strobilus $\sigma^{7}$ ellipsoideus ad subcylindricus, in sicco $15-17 \mathrm{~cm}$ longus, 4-4,5 cm diam., pedunculo ad 10$15 \mathrm{~mm}$ longo et $5 \mathrm{~mm}$ diam.; microsporophylla mediana $15-17 \mathrm{~mm}$ longa, 12-15 mm lata, ad axin sub angulo $75-80^{\circ}$ declivia, subsessilia; 
facies adaxialis concava, 1 vulticulo terminali rhomboideo, 2 vulticulis lateralibus trapezoideis et 1 vulticulo mediano rectangulari; facies abaxialis sporangiis operta, 2 vulticulis lateralibus inter se crista mediana bene notata. Strobilus $Q$ ovoideo-cylindricus, ad $1 / 3$ inferiore maxime inflatus, $29 \mathrm{~cm}$ longus et $15 \mathrm{~cm}$ latus pallido-viridis, pedunculo $1-1,5 \mathrm{~cm}$ longo et $4-4,5 \mathrm{~cm}$ diam.; megasporophylla mediana $6 \mathrm{~cm}$ lata, 1 vulticulo terminali rhomboideo, 2 vulticulis lateralibus trapezoideis, 1 vulticulo mediano triangulari cristis latero-sagittalibus obtusisque circumcincto.

ZAïre: Région du Shaba, route Kolwezi-Musokatanda km 27, à $24 \mathrm{~km}$ à l'WSW de Kolwezi, à $2 \mathrm{~km}$ au N-E de Mupenda, savane arbustive sur sables de Kalahari, alt. $1450 \mathrm{~m}$, mars 1988, Malaisse, Moretti \& Sclavo 2 (BR, O'); à $26 \mathrm{~km}$ à l'WSW de Kolwezi, Mupenda $\left(10^{\circ} 44^{\prime} \mathrm{S}, 27^{\circ} 15^{\prime} \mathrm{E}\right)$, savane sur sables de Kalahari, alt. $1475 \mathrm{~m}$, mars 1988, Malaisse, Moretti \& Sclavo 3 (BR, $Q$, holotype); à $32 \mathrm{~km}$ à l'WSW de Kolwezi, à 3,5 km à l'ESE de Ndjoni, forêt claire sur sables de Kalahari, alt. $1500 \mathrm{~m}$, mars 1988, Malaisse, Moretti \& Sclavo 4 (BR).

Habitat: Savanes arbustives diverses, notamment à Albizzia antunesiana, Terminalia sp. et Brachystegia spiciformis à proximité de savanes steppiques sur sables de Kalahari.

Nom vernaculaire: Makindu ya dilungu (dial. ndembo), signifie littéralement "palmier des savanes herbacées».

Observations: 1. - La plante est dédiée à Mr. Michel Schaijes dont les herborisations dans la région de Kolwezi ont fait progresser sensiblement la connaissance floristique de ce territoire.

2. - La fig. 7 présente l'état actuel des connaissances relatives à la distribution des Encephalartos en Afrique centrale.

Remerciements: Les auteurs remercient vivement Mr. le Dr. A. Robyns pour divers conseils et la mise au point de la description latine. Ils ont pu bénéficier de l'aide efficace de Kisimba Kibuye et Muzinga Yumba sur le terrain, qu'ils en soient remerciés.

Fig. 6. - Encephalartos schaijesii Malaisse, Sclavo \& Crosiers, strobiles mâle et femelle (d'après Malaisse, Moretti $\mathcal{E}$ Sclavo 3, dessins J. Defaij): A-C, strobile $\sigma^{\prime \prime}(\times 3 / 4)$; $\mathrm{A}$, aspect général du strobile; $\mathrm{B}$, détail de la bulla; $\mathrm{C}$, face abaxiale; $\mathrm{D}-\mathrm{F}$, strobile $O$ $(\times 5 / 11) ; D$, aspect général du strobile; $E$, détail de la bulla; $F$, face abaxiale. 

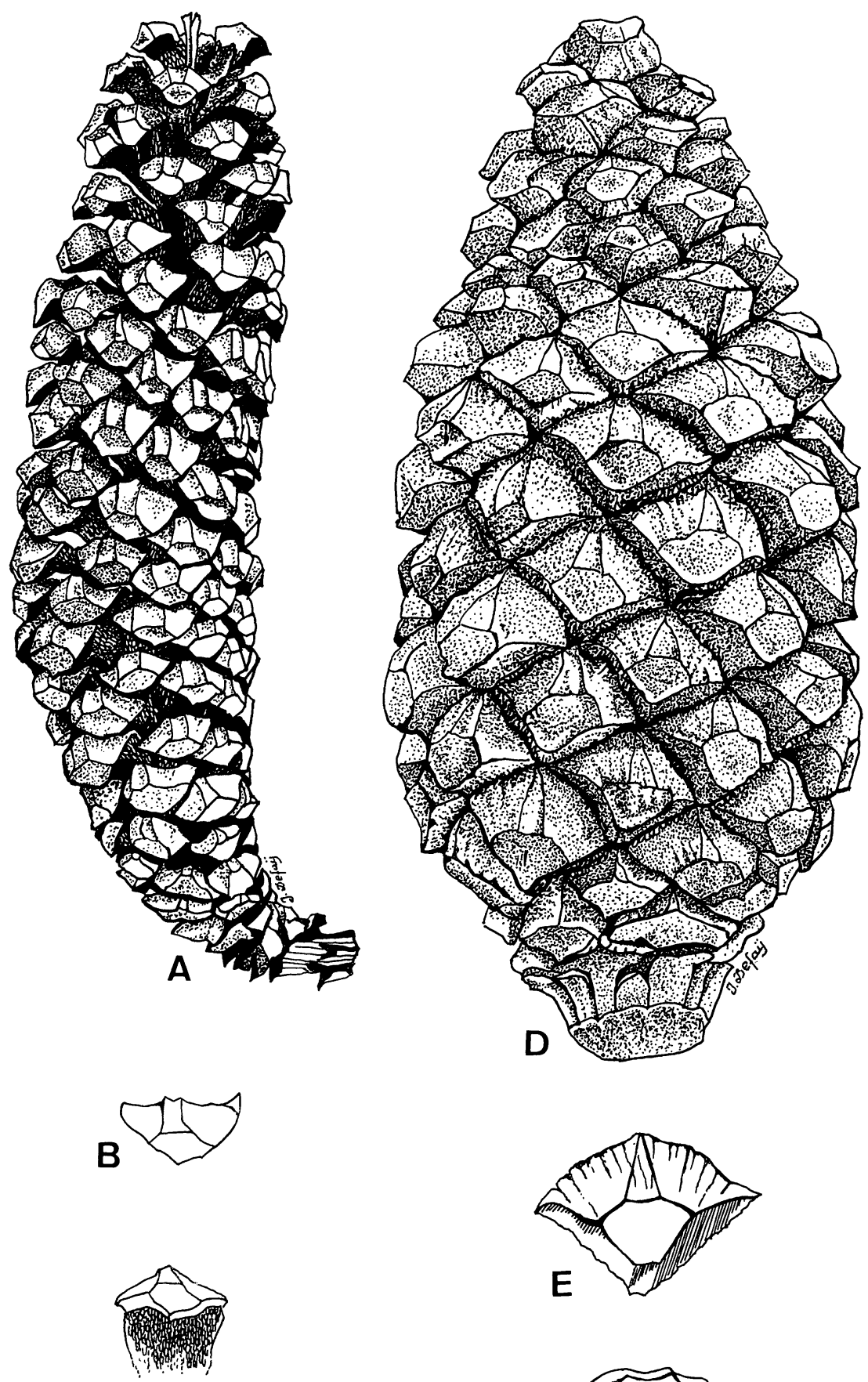

C

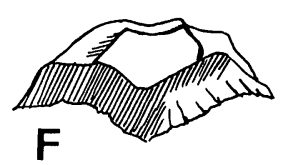




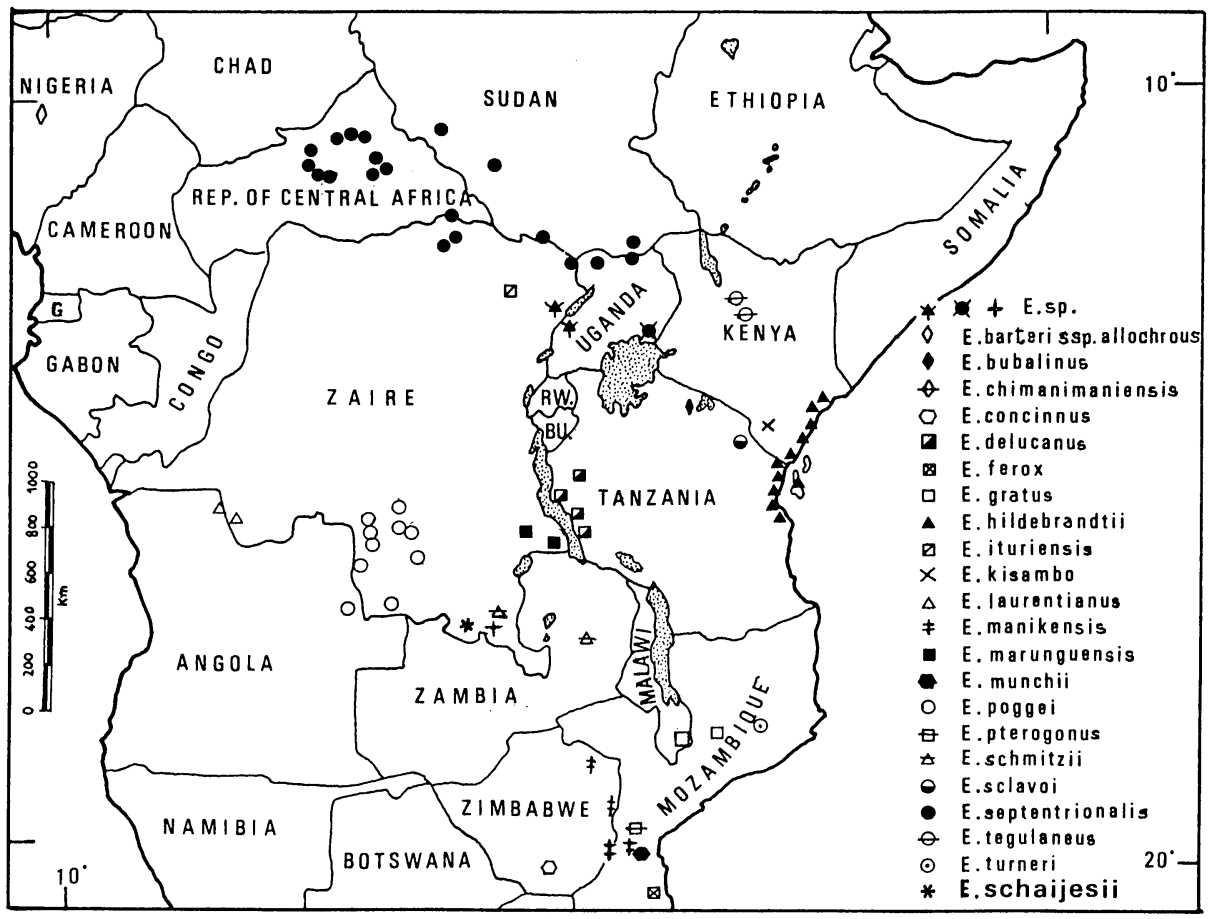

Fig. 7. - Carte de distribution des Encephalartos en Afrique centrale (d'après Melville 1957, Devred 1959, Moretti \& De Luca 1989, Stevenson et al. 1990 et Malaisse et al. 1990 modifié).

\section{REFERENCES}

Bamps P. \& Lisowski S. - (1990) A new species of Encephalartos (Zamiaceae) from Northeastern Zaire, in D.W. Stevenson (ed.), The Biology, Structure, and Systematics of the Cycadales. Mem. New York Bot. Gard. 57: 152-155.

De Luca P., Sabato S. \& Vazquez Torres M. (1982) - Distribution and variation of Dion edule (Zamiaceae). Brittonia $34: 355-362$.

Devred R. (1959) - Une Cycadacée nouvelle du Congo belge : Encephalartos marunguensis Devred. Bull. Soc. Roy. Bot. Belg. 91: 103-108.

Giddy C. (1984) - Cycads of South Africa, ed. 2 : 122 p. Cape Town, Struik.

Goode D. (1989) - Cycads of Africa : 256 p. Cape Town, Ed. J. Comrie-Greig, Struik. Heenan D. (1977) - Some observations on the cycads of Central Africa. Bot. Journ. Linn. Soc. London 74 : 279-288.

Koeleman A., Robbertse P.J. \& Eicker A. (1981) - Die anatomie van die pinnas van die suid-afrikaanse spesies van Encephalartos Lehm. Journ. S. Afr. Bot. 47: 247-271.

Lisowski S. \& Malaisse F. (1971) - Encephalartos marunguensis Devred, Cycadacée endémique du plateau des Muhila (Katanga, Congo-Kinshasa). Bull. Jard. Bot. Nat. 
Belg. 41 : 357-361.

Malaisse F. (1969) - Encephalartos schmitzii Malaisse, Cycadacée nouvelle du CongoKinshasa. Bull. Jard. Bot. Nat. Belg. 39: 401-406.

Malaisse F., Sclavo J.P. \& Turner I. (1990) - Zamiaceae, a new family for Zambia, in D.W. Stevenson (ed.), The Biology, Structure, and Systematics of the Cycadales. Mem. New York Bot. Gard. 57 : 162-168.

Melville R. (1957) - Encephalartos in Central Africa. Kew Bull. $1957:$ 237-257.

Moretti A., De Luca P., Sclavo J.P. \& Stevenson D. (1989) - Encephalartos voiensis (Zamiaceae), a new East Central African species in the E. hildebrandtii complex. Ann. Missouri Bot. Gard. 76 : 934-938.

Stevenson D.W., Moretti A. \& De Luca, P. (1990) - A new species of Encephalartos (Zamiaceae) from Tanzania, in D.W. Stevenson (ed.), The Biology, Structure, and Systematics of the Cycadales. Mem. New York Bot. Gard. 57: 156-161. 